\title{
Polysaccharide-Based Lotus Seedpod Surface-Like Porous Microsphere as an Efficient Drug Carrier for Cancer Treatment
}

\author{
Yuanbo $\mathrm{Wu}^{1, *}$ \\ Jiandong Zhang ${ }^{2, *}$ \\ Jiangwei $\mathrm{Ni}^{2}$ \\ Zhihao Yang (D) ${ }^{2}$ \\ Kun Chen $\mathbb{1 D}^{2}$ \\ Liangcheng Zheng ${ }^{2}$ \\ Zhifeng $\mathrm{He}^{2}$ \\ 'Department of Cardiothoracic Surgery, \\ The First Affiliated Hospital of Wenzhou \\ Medical University, Wenzhou, People's \\ Republic of China; ${ }^{2}$ Department of \\ Thoracic Surgery, The First Affiliated \\ Hospital of Wenzhou Medical University, \\ Wenzhou, People's Republic of China
}

*These authors contributed equally to this work
Correspondence: Liangcheng Zheng;

Zhifeng He

Email dr.zlc@163.com

zhif_he@I26.com
Background: This study aimed to evaluate the properties and functions of polysaccharidebased porous microsphere (PPM) for drug delivery, as well as its inhibitory effect on malignant tumors.

Materials and Methods: PPM was prepared using the inverse emulsion polymerization method. FT-IR measurements were conducted to measure the wavenumber of PPM. Particle size distribution was tested with a particle analyzer, and surface morphologies of PPM were observed using a scanning electron microscope (SEM). Dialysis method, Cell Counting Kit-8 (CCK-8), and cell apoptosis analysis were adopted to evaluate the drug release, cytotoxicity and biocompatibility of mitomycin-C (MMC), respectively. Finally, an in vivo study was performed in C57BL/6 mice to confirm the function of MMC-loaded PPM on tumor growth. Results: FT-IR spectra proved the successful preparation of MMC-loaded PPM. PPM had an average size of $25.90 \pm 0.34 \mu \mathrm{m}$ and then increased to $30.10 \pm 0.20 \mu \mathrm{m}$ after drug loading. Under SEM, the surface morphology was lotus seedpod surface-like, with macropits on the surface and micropores in macropits. Compared with the free MMC group, MMC-loaded PPM exhibited a delayed drug release rate in a $\mathrm{pH}$-dependent manner and higher cell viability. Flow cytometry results showed that the cell apoptosis in the PPM/MMC group was lower than that in the free MMC group. In vivo experiment revealed the inhibitory efficacy of MMC-loaded PPM on malignant tumors.

Conclusion: In summary, MMC-loaded PPM exhibited favorable surface morphology, sustained drug release ability, nontoxicity and excellent biocompatibility, suggesting that PPM might be a potential drug carrier for tumor treatment.

Keywords: polysaccharide-based porous microsphere, tumor treatment, mitomycin-C, drug release, inverse emulsion polymerization

\section{Introduction}

Cancer is the leading cause of death worldwide owing to its rapid growth, strong metastatic ability and high recurrence rate. ${ }^{1}$ At present, the traditional treatments for cancer mainly include chemotherapy, radiotherapy and surgery. ${ }^{2}$ It has been realized that most cancer patients are diagnosed at an advanced stage, making radical surgery impossible and treatment ineffective. ${ }^{3}$ Different from radiotherapy and surgical resection, chemotherapy can achieve systemic blood circulation of drugs through oral administration, intravenous injection and targeted delivery system, thereby achieving the effect of systemic treatment. $^{4,5}$ 
Mitomycin-C (MMC) is a commonly used anti-tumor drug, which plays an antagonistic role in deoxyribonucleic acid (DNA) replication after intracellular activation by reductase. ${ }^{6}$ A high concentration of MMC can inhibit the synthesis of RNA and protein. ${ }^{7}$ As a typical periodic nonspecific drug, MMC has been widely applied in the treatment of digestive tract cancers, such as gastric, cervical, bladder and lung cancers. ${ }^{8,9}$ However, due to the lack of tumor specificity, chemotherapy drugs not only kill tumor cells but also damage normal cells, thus shortening their circulation in blood compartments. Insufficient intratumoral retention of therapeutic agents and multidrug resistance are the major factors responsible for treatment failure in solid tumors. ${ }^{10}$

Currently, the application of drug carriers in traditional chemotherapy, such as micelles, carbon nanotubes, nanogels, dendritic macromolecules and microspheres, can effectively prolong blood circulation and promote drug absorption in tumor sites, thereby enhancing the therapeutic effect and safety of traditional chemotherapy. ${ }^{11-13}$ Gharbavi et al found that, as a tamoxifen tumor-targeted delivery system, cholesterol conjugated bovine serum albumin nanoparticles exhibited high toxicity against mouse breast cell lines. ${ }^{14}$ In addition, Ashrafizadeh et al demonstrated the high efficiency of nano-carriers in the delivery of siRNA in prostate cancer therapy. ${ }^{15}$

Polysaccharides refer to a multiple class of natural polymeric materials that derive from animals, plants and alga. ${ }^{16}$ The molecular weight of natural polysaccharide ranges from hundreds to thousands of Daltons, which further increases their diversity. It has been reported that polysaccharides are involved in immunomodulation, antibacterial and pharmaceutical applications because of their economical, easily available, nontoxic and biodegradable properties. ${ }^{13}$ Meanwhile, as nontoxic chemo preventive or chemotherapeutic agents, polysaccharides have received extensive attention in relation to their capability to induce tumor cell death and inhibit gene expression in a variety of cancers. ${ }^{17}$ In the field of drug carriers, polysaccharidebased microsphere polymers have abundant reserves, with low cost and easy modification. Shu et al demonstrated that polysaccharide-based microsphere polymers possess excellent biocompatibility, biodegradability and biological adhesion, making them ideal drug carrier matrices for immune regulation, anti-tumor and antibacterial effects and drug delivery. ${ }^{18}$

In this paper, a polysaccharide-based porous microsphere (PPM) with a lotus seedpod surface-like structure was designed, and its efficacy in drug delivery and antitumor effects was further evaluated.

\section{Materials and Methods Materials}

Sixteen healthy C57BL/6 nude mice (male, 4-6 wks old, weighing 20-30 g) were obtained from the Experimental Animal Center of Wenzhou Medical University (SYXK2015-0009). Carboxymethyl chitosan (Mw = 150,000-200,000, carboxyl proportion: $\geq 80 \%$ ) was obtained from Shanghai yuanye Bio-Technology Co., Ltd (Shanghai, China). Sodium alginate $(\eta=20020 \mathrm{mp} \cdot \mathrm{s})$, Span-80, and PBS were provided by Aladdin Biological Technology Co., Ltd (Shanghai, China). Calcium chloride $\left(\mathrm{CaCl}_{2}\right)(99 \%)$ was supplied by Macklin Biochemical Science and Technology Co., Ltd. (Shanghai, China). Cell Counting Kit-8, A549 cells, Annexin V-FITC and PI were all purchased from Beyotime Biotechnology Co., Ltd (Shanghai, China).

\section{Preparation of PPMs and Drug Loading}

PPMs were prepared using the inverse emulsion polymerization method, as shown in Figure 1. Carboxymethyl chitosan and sodium alginate were used as the raw material of polysaccharides, and $\mathrm{CaCl}_{2}$ solution was used as the crosslinker, as previously described. ${ }^{19}$ Firstly, $90 \mathrm{~g}$ of paraffin oil was added into a three-necked flask, followed by the addition of Span- $80(0.4 \%, \mathrm{w} / \mathrm{w})$ as the emulsifier. Then, the mixture was emulsified for $1 \mathrm{~h}$ by stirring at 200-300 rpm under gentle magnetic agitation. After mixing with $100 \mathrm{~mL}$ of distilled water, the $2.5 \%(\mathrm{w} / \mathrm{w}) \mathrm{mix}-$ ture solution of carboxymethyl chitosan/sodium alginate was used as a water phase. At $30^{\circ} \mathrm{C}, 30 \mathrm{~mL}$ of water phase was dropwise added to oil phase, and then a homogeneous and stable microemulsion was formed after stirring for 2 $\mathrm{h}$ at $700 \mathrm{rpm}$. Subsequently, the cross-linking reaction was achieved after the addition of $\mathrm{CaCl}_{2}$ solution $(20 \mathrm{wt} \%)$ to the microemulsion, followed by a continuous $4 \mathrm{~h}$ reaction at $30^{\circ} \mathrm{C}(200-300 \mathrm{rpm})$. PPMs were acquired by washing by hexane, ethanol and water, respectively. Finally, PPMs were dried in a vacuum oven at $40^{\circ} \mathrm{C}$ and then kept in a vacuum desiccator for later use.

MMC was selected as the model drug for drug loading. Due to the good adsorption capacity of PPM, free MMC and PPMs were mixed in water, and the MMC was absorbed within $5 \mathrm{~min}$. Thus, MMC-loaded PPM was established successfully. 


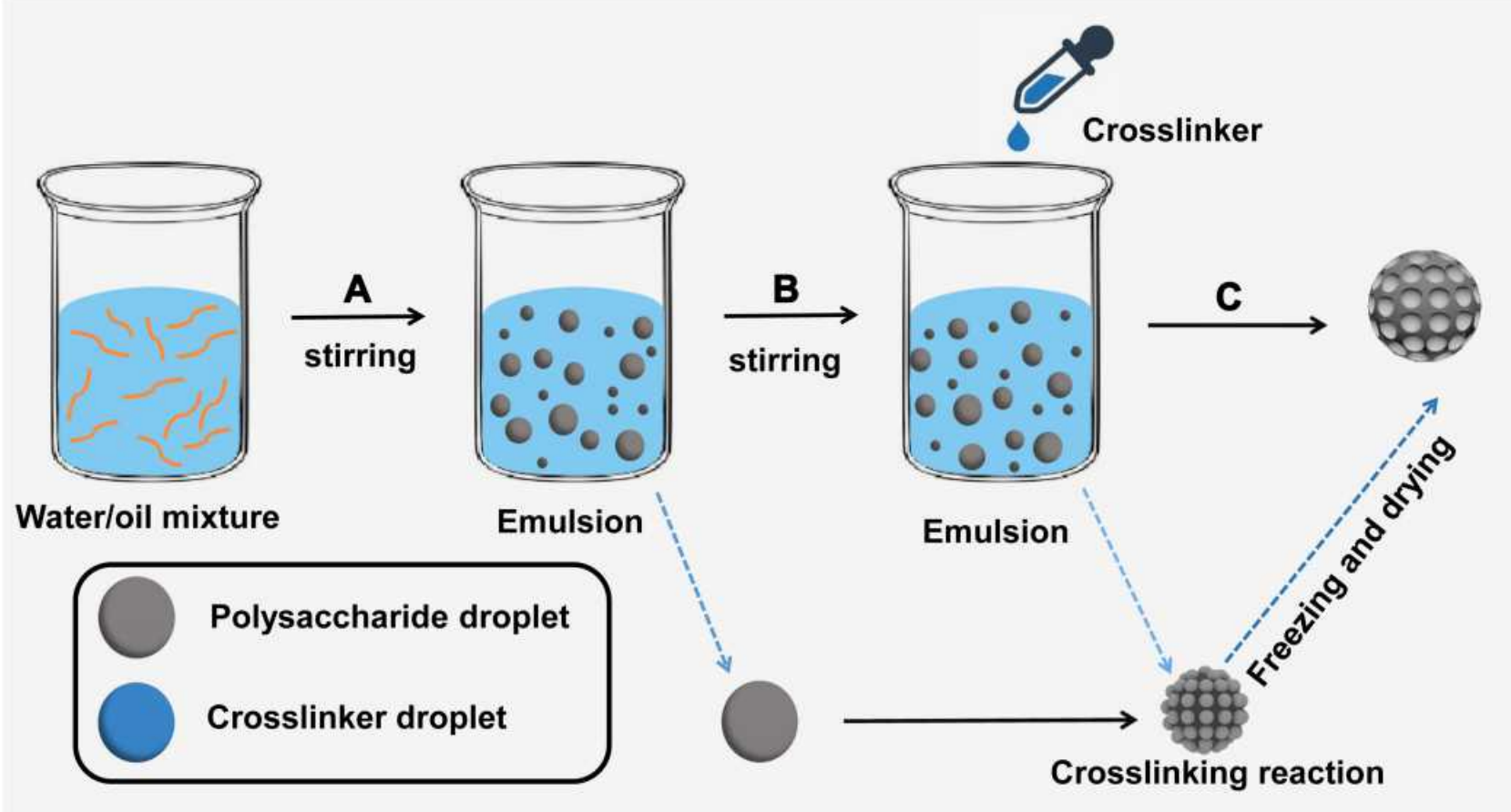

Figure I PPM synthesis process diagram. (A) Water and oil mixture were stirred to form inverse emulsion. Polysaccharide large emulsion droplets were evenly dispersed in the oil phase. (B) The crosslinker $\mathrm{CaCl}_{2}$ solution was added to the emulsion and formed tiny emulsion droplets. A crosslinking reaction was performed between polysaccharide droplets and tiny crosslinker droplets. (C) After freezing and drying, PPMs were finally obtained.

\section{FT-IR Spectroscopy}

Fourier transform infrared (FT-IR) measurements were carried out using FT-IR spectrograph (Tensor II, Bruker, Germany) in the wavenumber range of $3600-400 \mathrm{~cm}^{-1}$. FT-IR spectra were acquired from PPMs and MMC-loaded PPM.

\section{Morphology, Size Distribution and Zeta Potential}

The surface morphologies of PPMs were observed by a scanning electron microscope (SEM, SU8010, HITACHI, Japan) at $20 \mathrm{kV}$. A particle analyzer (Zetasizer Nano ZS90, Malvern, UK) was used to determine the zeta potential and particle size distribution of PPM and MMC-loaded PPM.

\section{Drug Release Rate in vitro}

The dialysis method was performed to measure the in vitro release of free MMC and MMC-loaded PPM at pH 6.5 and 7.4. PBS at $\mathrm{pH} 6.5$ and 7.4 was selected as the releasing medium. The MMC-loaded PPM and free MMC were added into PBS $10 \mathrm{~mL}$ for dispersion, respectively. Then, $2 \mathrm{~mL}$ of the dispersion was added into a dialysis bag, followed by centrifugation at $37^{\circ} \mathrm{C}$ at $60 \mathrm{rpm} / \mathrm{min}$. Finally, the HPLC method was used to determine the drug content in the release solution and calculate the cumulative release percentage (\%).

\section{CCK-8 Assay}

CCK-8 assay was adopted to evaluate the cytotoxicity of MMC-loaded PPM to A549 cells. A549 cells were seeded in a 96-well cell culture plate, with $4 \times 10^{3}$ cells per well. After incubation for $24 \mathrm{~h}$, the culture medium was replaced by a series of concentrations of MMC $(0,0.125$, $0.5,0.75,1$ and $1.5 \mu \mathrm{g} / \mathrm{mL}$ ) diluted with the corresponding culture fluid. Three replicates were made for measurements of free MMC, MMC-loaded PPM and free PPM. After co-incubation for $48 \mathrm{~h}, 10 \mu \mathrm{L}$ of CCK-8 reagent was added to each well. Then, OD at $450 \mathrm{~nm}$ was measured using a microplate reader (Infinite M200 Pro, Tecan) after incubation for $2 \mathrm{~h}$ at $37^{\circ} \mathrm{C}$.

\section{Flow Cytometry}

The biocompatibility and cytotoxicity of the drug carrier on A549 cells were assessed in this study. Firstly, the cells were washed twice with PBS and centrifuged at 1,000 rpm for $5 \mathrm{~min}$. After the cells were re-suspended in $300 \mu \mathrm{L}$ of 
binding buffer, different concentrations of the target drugs were added. The cells and drugs were co-cultured for 24 h. Then, $5 \mu \mathrm{L}$ of Annexin V-FITC and $5 \mu \mathrm{L}$ of PI were added and incubated for another $10 \mathrm{~min}$, followed by the addition of $200 \mu \mathrm{L}$ of binding buffer. Cell apoptosis analysis was performed by flow cytometry (ACEA Biosciences Inc.).

\section{In vivo Antitumor Assay}

All mouse experiments were approved by the Ethics Committee of the First Affiliated Hospital of Wenzhou Medical University (wydw2020-0509) and performed under the guidelines of the Institutional Animal Care and Use Committee (IACUC). Sixteen healthy C57BL/6 nude mice were fed with purified water and commercial stock diet in an air-conditioned room with regular UV disinfection. Firstly, the cell suspension was obtained by digesting A549 cells at the logarithmic growth stage with $0.25 \%$ trypsin. Subsequently, the cell suspension was mingled with the same amount of Gel Matrix to make the cell mixture. Next, $2 \times 10^{6}$ A549 cells were injected subcutaneously into the right shoulder of each nude mouse. After solid tumors grew to about $100 \mathrm{~mm}^{3}$, nude mice were divided into control group (saline), MMC group, PPM group and PPM/MMC group, with 4 mice per group. The drug was sterilized by passing through a $0.22 \mu \mathrm{m}$ membrane filter and injected daily by tail vein (i.v.). Tumor volumes were measured and recorded by a Vernier caliper starting from the 7th day after tumor inoculation. All mice were sacrificed on the 21st day and the solid tumors were dissected and weighed.

\section{Statistical Analysis}

All data were analyzed using SPSS15.0 statistical software. Comparison between different experimental groups was performed with the Student's $t$-test or one-way analysis of variance (ANOVA) with Dunnett's test. Statistical significance for pairwise comparison was determined using two-way ANOVA with Tukey test. A value of $p<0.05$ was considered statistically significant.

\section{Results}

\section{Characterization of PPMs and MMC-Loaded PPM}

After the formation of the microsphere, PPM and MMCloaded PPM were subjected to FT-IR measurements. Absorption peaks at $3305 \mathrm{~cm}^{-1}$ were ascribed to $\mathrm{O}-\mathrm{H}$ on
PPM. Absorption peaks at 2920 and $2870 \mathrm{~cm}^{-1}$ were mainly owing to the $\mathrm{C}-\mathrm{H}$ bond on the PPM molecular chain. The characteristic peaks at $1590 \mathrm{~cm}^{-1}$ of both spectra were the carbonyl group $(\mathrm{C}=\mathrm{O})$ on the PPM molecular chain. The absorption peaks at $1310 \mathrm{~cm}^{-1}$ were assigned to the C-N bond on the PPM molecular chain (Figure 2). These findings revealed that MMC was successfully loaded into the PPM.

\section{Morphology and Particle Size}

As shown in Table 1, the average size of PPM increased from $25.90 \pm 0.34 \mu \mathrm{m}$ to $30.10 \pm 0.20 \mu \mathrm{m}$ after drug loading, indicating that the absorption of MMC increased particle size. In addition, the zeta potentials of PPM before and after drug loading did not change significantly $(-13.21$ $\pm 0.10 \mathrm{mV}$ vs $-14.20 \pm 0.30 \mathrm{mV})$. The morphology of the lotus seedpod surface-like PPM under SEM further supported the findings of the particle analyzer, showing many macropits on the surface and micropores in macropits (Figure 3A). Moreover, after drug loading, there were small particles adhering to the pores on the blurred surface of the microspheres, revealing that MMC was successfully loaded into PPM (Figure 3B).

\section{Drug Release Rate in vitro}

As shown in Figure 4A, the release rates of MMC in free $\mathrm{MMC}$ and PPM/MMC groups were $\mathrm{pH}$-dependent, and more rapid release was observed at $\mathrm{pH} 6.5$ rather than at $\mathrm{pH}$ 7.4. At the corresponding $\mathrm{pH}$, the release rates of $\mathrm{MMC}$ in the PPM/MMC group were lower than those in the free MMC group at $\mathrm{pH} 6.5$ and $\mathrm{pH} 7.4$ (both $p<0.01$ ). These data indicated that free MMC and MMC-loaded

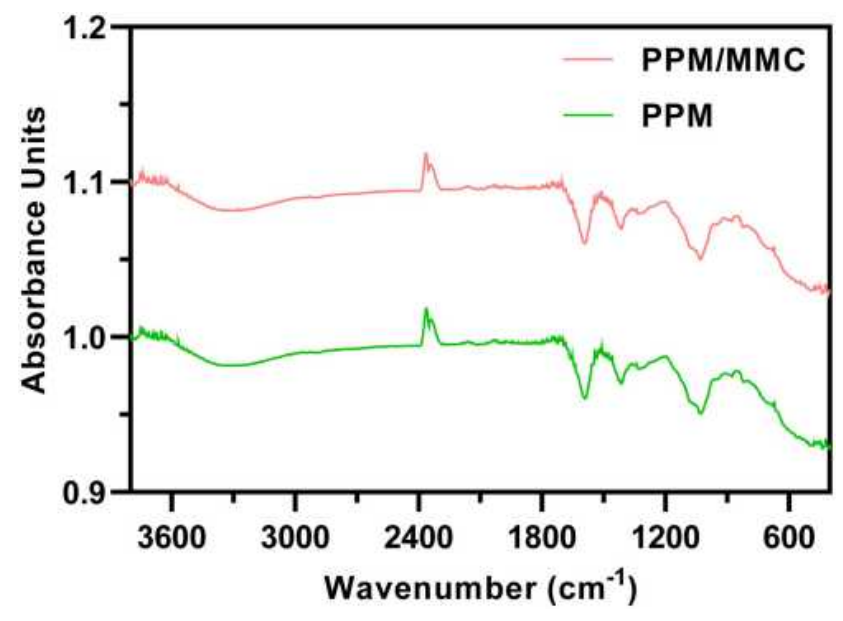

Figure 2 Fourier transform infrared (FT-IR) spectroscopy of PPM and PPM/MMC (MMC-loaded PPM). 
Table I Average Size and Zeta Potential of PPM and MMCLoaded PPM Obtained by Particle Analyzer

\begin{tabular}{|l|c|c|}
\hline Materials & Average Size $(\boldsymbol{\mu m})$ & Zeta Potential $(\mathbf{m V})$ \\
\hline PPM & $25.90 \pm 0.34$ & $-13.21 \pm 0.10$ \\
PPM/MMC & $30.10 \pm 0.20$ & $-14.20 \pm 0.30$ \\
\hline
\end{tabular}

Note: Mean \pm SEM (standard error of mean).

Abbreviations: PPM, polysaccharide-based porous microsphere; MMC, mitomycin-C.

PPM released more rapidly under acidic conditions; furthermore, MMC encapsulated in PPM released less.

\section{MMC-Loaded PPM Reduced Cytotoxicity and Apoptosis of A549 Cells}

The viability of A549 cells in the presence of free MMC, PPM and MMC-loaded PPM was assessed by CCK-8 assay to determine the potency of PPM. As shown in Figure $4 \mathrm{~B}$, a considerable reduction in cell viability was found when A549 cells were treated with free MMC, indicating that free MMC showed significant toxicity towards A549 cells $(p<0.0001)$. In contrast, PPM alone had no obvious toxicity on A549 cells, with high cell viability, which revealed great biocompatibility of PPM. Compared with the free MMC group, cell viability in the PPM/MMC group was significantly promoted $(p<0.01)$.

The apoptosis rate of A549 cells in the PPM group was slightly reduced when compared with the free
MMC and PPM/MMC groups, implying the nontoxicity and excellent biocompatibility of PPM. Meanwhile, there was less cell apoptosis in the PPM/MMC group than in the free MMC group (Figure 5). Collectively, these data indicated that PPM could significantly reduce the toxicity of MMC, which might be associated with the slow-release function of PPM.

\section{Antitumor Efficacy of MMC-Loaded PPM in vivo}

To evaluate the function of PPM/MMC on tumor growth, C57BL/6 nude mice were subjected to in vivo experiments. Results showed that there was no significant difference in tumor volume between the 4 groups within $11 \mathrm{~d}$ after administration. Nevertheless, tumor volume in the PPM/MMC group was significantly smaller than that in the free MMC group by day $21(p<0.001)$ (Figures 6A and B). In addition, mice treated with MMC-loaded PPM weighed less than than those treated with free MMC alone $(p<0.001)$ (Figure 6C). Overall, PPM significantly enhanced the efficacy of MMC, and the MMC-loaded PPM had a potent inhibitory action on solid tumor.

\section{Discussion}

In recent decades, researchers have focused on a variety of carriers with varying degrees of efficacy and drawbacks, including nanocapsules, microspheres and micelles. ${ }^{11-13}$

\section{A}

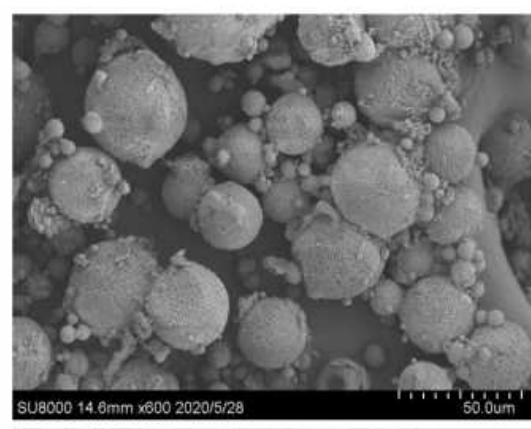

B

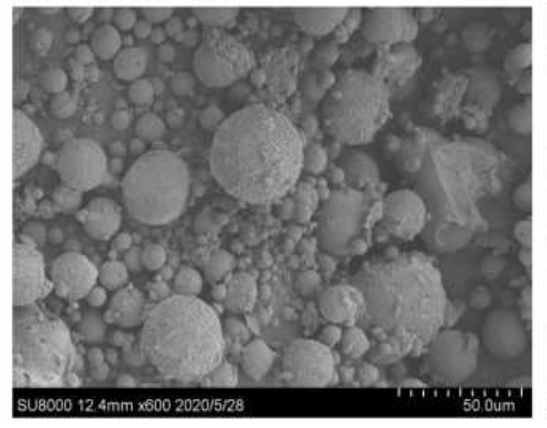

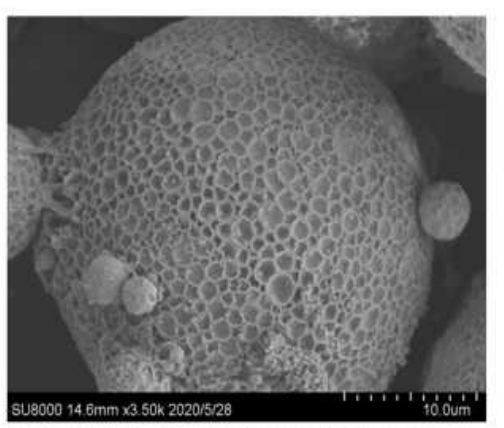

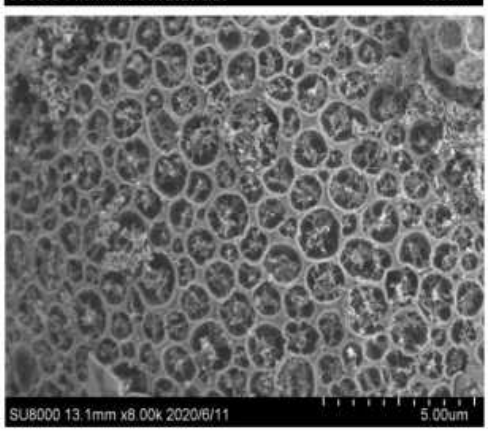

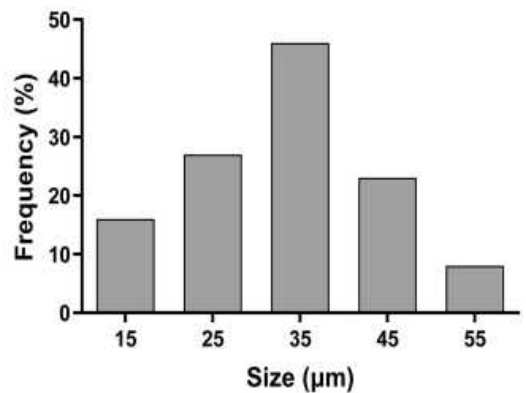

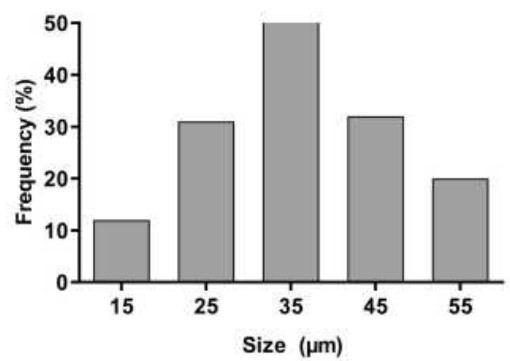

Figure 3 Scanning electron microscope analysis morphology of (A) PPM and (B) MMC-loaded PPM. 

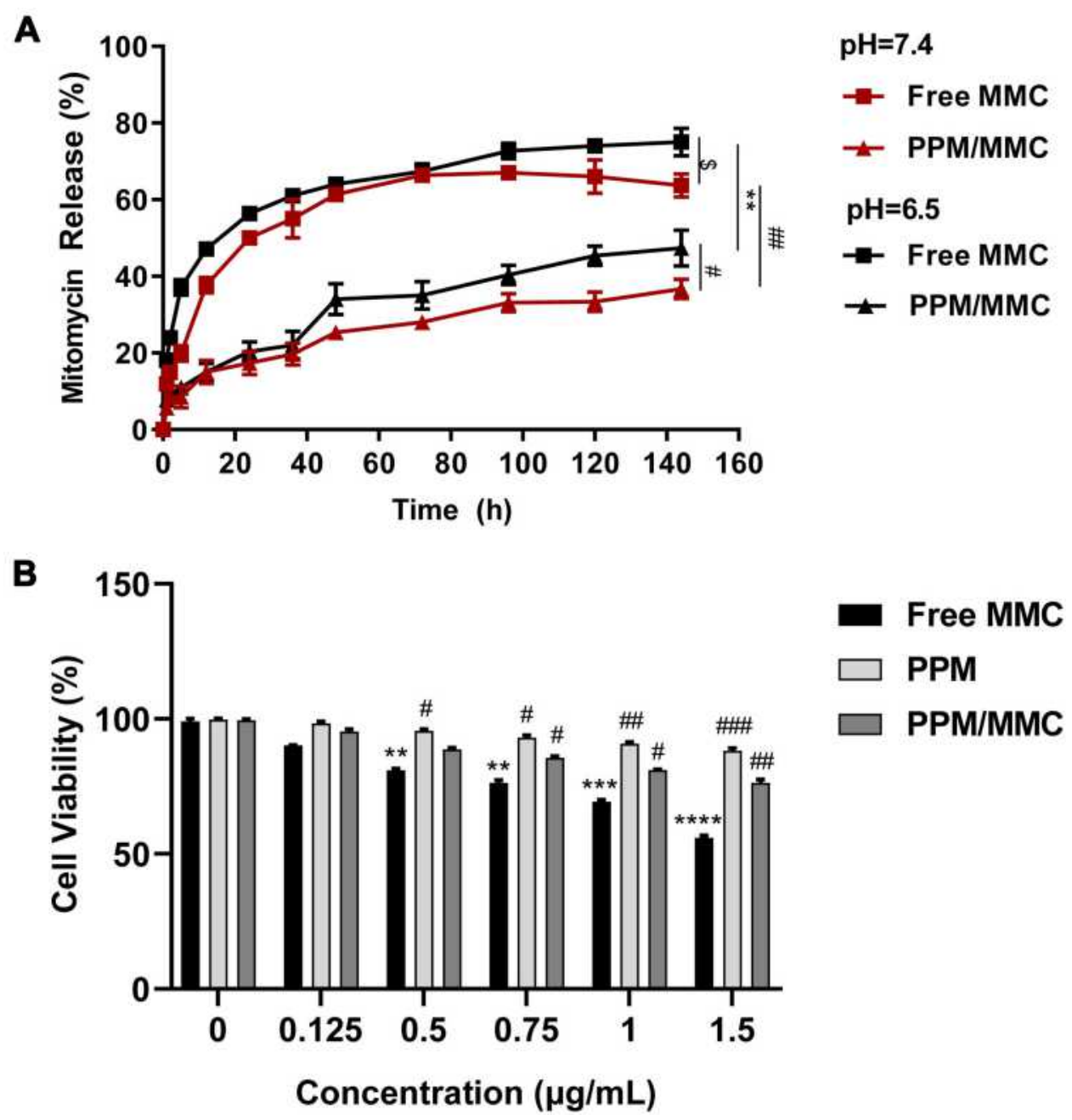

Figure 4 (A) Drug release results of the free MMC and MMC-loaded PPM at different $p H .{ }_{p}<0.05$, compared with free $M M C$ group at $p H$ 6.5. $* * p<0.01$, compared with free MMC group at $\mathrm{pH} 6.5 .{ }^{\#} p<0.05$, compared with $\mathrm{PPM} / \mathrm{MMC}$ group at $\mathrm{pH} 6.5$; ${ }^{\#} p<0.0 \mathrm{I}$, compared with free MMC group at $\mathrm{pH} 7.4$. (B) Cell viability rate of $\mathrm{A} 549$ cocultured with free MMC, MMC-loaded PPM and free PPM at different concentrations for $24 \mathrm{~h}$ was assessed by $C C K-8$. $* * p<0.0 \mathrm{I}$, $* * * p<0.00 \mathrm{I}$ and $* * * * p<0.000 \mathrm{I}$, compared with control group $(0 \mu \mathrm{g} / \mathrm{mL}) ;{ }^{\#} p<0.05,{ }^{\#} p<0.01$ and ${ }^{\# \#} p<0.00$ I, compared with free MMC group.

$\mathrm{Yu}$ et $\mathrm{al}^{20}$ fabricated proanthocyanidins/chitosan/lecithin microspheres by spray-drying technology and confirmed that this microsphere could serve as a pulmonary drug carrier with desirable moisture content, tapped density and moisture uptake. De-esterified tragacanth microspheres loaded into Eudragit S-100 coated capsules have also been shown to be effective for colon-targeted delivery. ${ }^{21}$ The current study described a successful preparation of polysaccharide-based porous microsphere via the inverse emulsion polymerization method, with carboxymethyl chitosan and sodium alginate as the raw material of polysaccharides, $\mathrm{CaCl}_{2}$ solution as the crosslinker and $\mathrm{MMC}$ as the model drug. In vitro study revealed that MMC-loaded PPM possessed excellent biocompatibility and sustained drug release, accompanied by high-drug loading capacity. Moreover, in vivo experiments indicated an intrinsic anti-tumor effect of MMC-loaded PPM.

Polymers have been used as novel drug carriers in the field of drug targeting and delivery because of their high drug-loading capacity and unique disposition characteristics in the body. ${ }^{22}$ Chitosan, a natural polymer obtained by alkaline deacetylation of chitin, exhibits excellent nontoxic, biocompatible and biodegradable properties. A study conducted by Parhi et al showed that chitosan is a suitable material for efficient drug delivery due to its enhanced target-specific drug release and drug bioavailability. $^{23}$ In our work, PPM was synthesized based on chitosan via the inverse emulsion polymerization method, which was easy to control and operate, and the drug loading was achieved by physical absorption. $\mathrm{Xu}$ et al pointed out that particles with diameters less than 
A

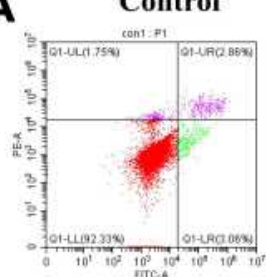

$0.75 \mu \mathrm{g} / \mathrm{mL}$

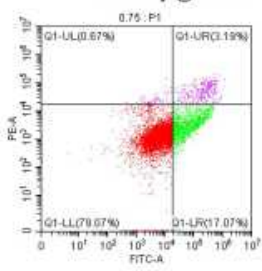

B

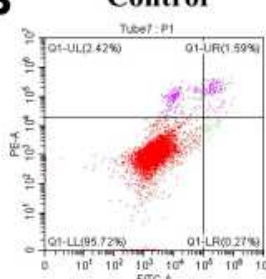

$0.75 \mu \mathrm{g} / \mathrm{mL}$
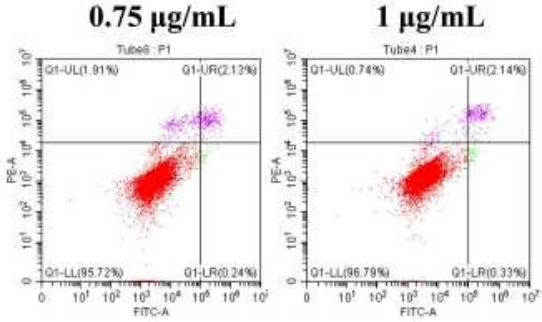

$0.125 \mu \mathrm{g} / \mathrm{mL}$

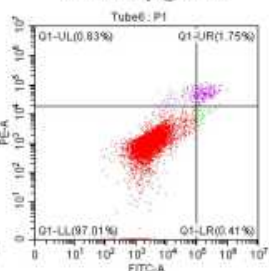

$1 \mu \mathrm{g} / \mathrm{mL}$

C

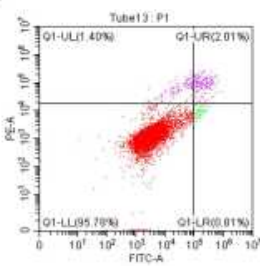

$0.75 \mu \mathrm{g} / \mathrm{mL}$

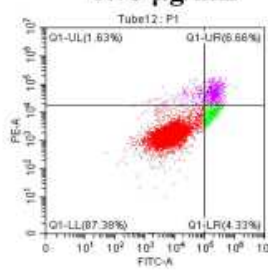

$0.125 \mu \mathrm{g} / \mathrm{mL}$

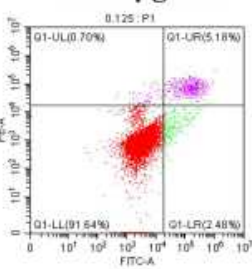

$1 \mu \mathrm{g} / \mathrm{mL}$

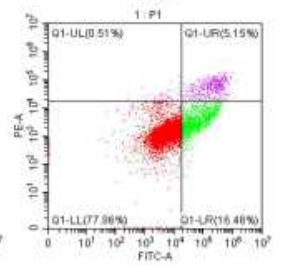

$0.125 \mu \mathrm{g} / \mathrm{mL}$

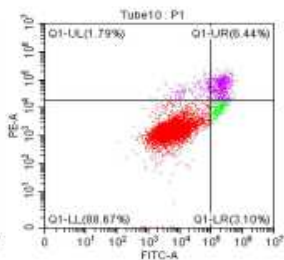

$1 \mu \mathrm{g} / \mathrm{mL}$

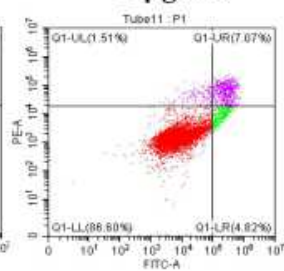

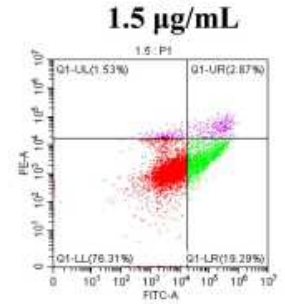

$0.5 \mu \mathrm{g} / \mathrm{mL}$

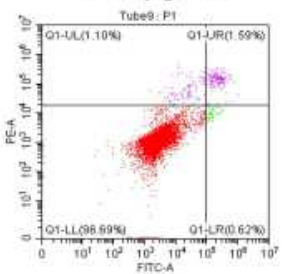

$1.5 \mu \mathrm{g} / \mathrm{mL}$

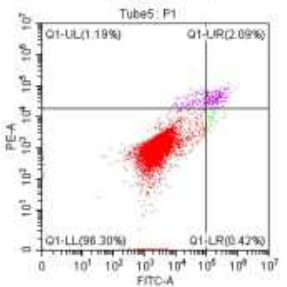

$0.5 \mu \mathrm{g} / \mathrm{mL}$

$0.5 \mu \mathrm{g} / \mathrm{mL}$

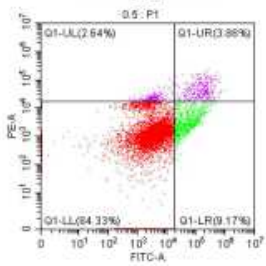

$1.5 \mu \mathrm{g} / \mathrm{mL}$
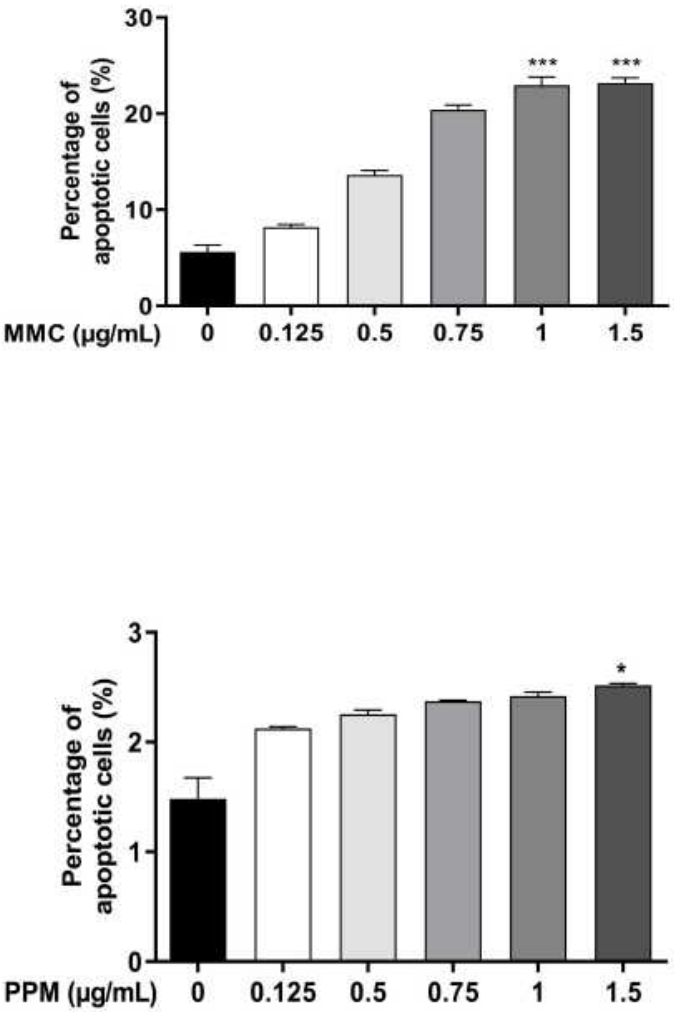

Figure 5 (A) Apoptosis experiment results of A549 co-cultured with free MMC for $24 \mathrm{~h}$ at different concentrations. (B) Apoptosis experiment results of A549 co-cultured with PPM alone for $24 \mathrm{~h}$ at different concentrations. (C) Apoptosis experiment results of A549 co-cultured with MMC-loaded PPM for $24 \mathrm{~h}$ at different concentrations. $* p<0.05$ and $* * * p<0.001$, compared with control group.

$80 \mathrm{~nm}$ have a longer circulation time than larger particles. $^{12}$ In the present study, PPM had an average size of $25.90 \pm 0.34 \mu \mathrm{m}$, and then increased to $30.10 \pm 0.20$ $\mu \mathrm{m}$ after drug loading, which was within the range of previous studies. SEM results clarified the presence of unique macropits on the surface morphology and 


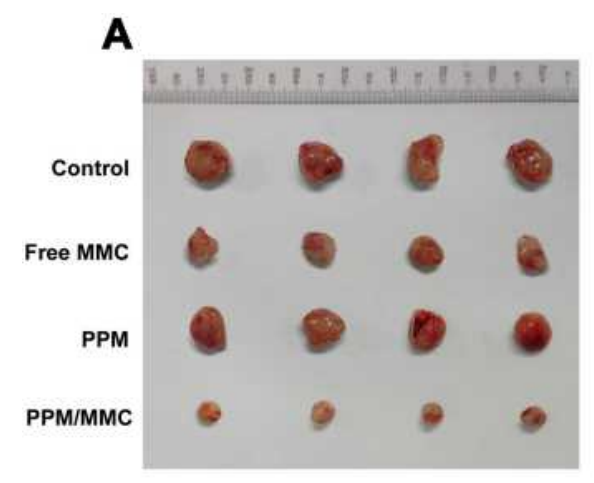

B
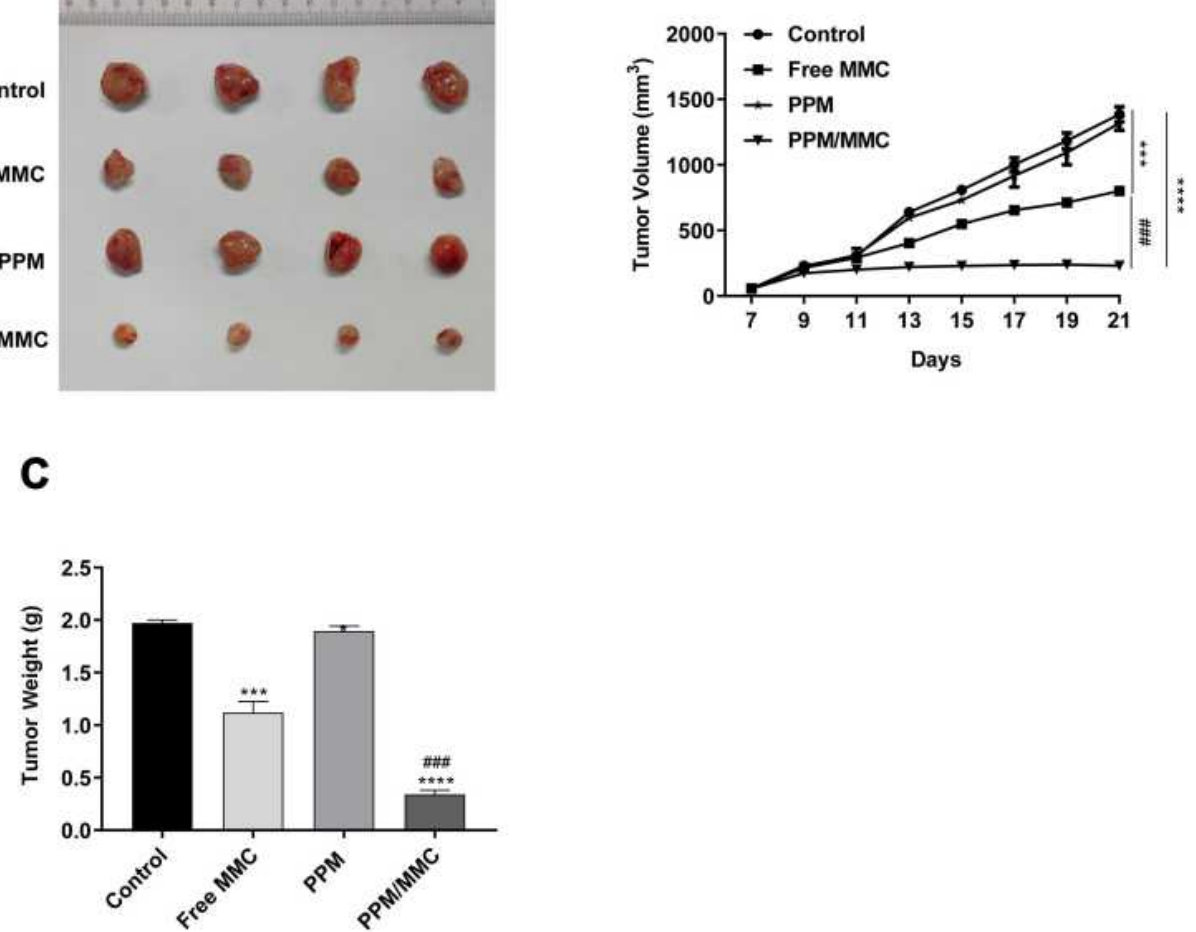
and MMC-loaded PPM) ( $n=4$ per group). (C) Tumor weight of mice was determined after different treatments. ${ }^{*} * *_{p}<0.001$ and $* * * * p<0.0001$, compared with control group.

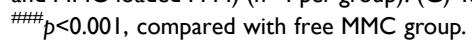

micropores in the macropits structure of PPM, namely, lotus seedpod surface-like PPM.

The efficacy of traditional chemotherapy in cancer treatment may be limited by systemic toxicity of chemotherapeutic agents. ${ }^{14}$ As one of the potent anticancer agents, MMC can kill cancer cells while debilitating patients. Microspheres can reduce drug distribution in normal organs, improve efficiency and reduce toxicity. ${ }^{20}$ In this research, CCK-8 analysis showed that PPM/MMC significantly inhibited the viability of A549 cells. According to the cell apoptosis analysis, the PPM/MMC group showed lower cell apoptosis than the free MMC group, partially due to controlled drug release by PPM. When loaded by PPM, MMC in the medium was controlled at a lower concentration, implying that PPM was able to diminish the cytotoxicity of MMC and alleviate the discomfort in cancer treatment. A recent study showed that $\mathrm{pH}$ value can regulate drug release rate, with a more acidic $\mathrm{pH}$ value releasing the microsphere-entrapped drugs more quickly. $^{24}$ In the current study, the drug release rate of MMC-loaded PPM at $\mathrm{pH} 6.5$ was faster than that at $\mathrm{pH}$ 7.4, suggesting that MMC-loaded PPM had potential for tumor targeting.
A549 cells were injected subcutaneously into C57BL/6 nude mice to detect the anti-tumor function of MMCloaded PPM on tumor growth in vivo. At day 21, mice treated with MMC-loaded PPM exhibited smaller tumor volume than those treated with free MMC. In addition, compared with the free MMC group, PPM/MMC significantly lowered tumor weight. As previous investigations have shown, the combination of polysaccharide D-Fraction extracted from maitake mushrooms with MMC can enhance the outcome and quality of life of cancer patients, ${ }^{25}$ which may explain the strengthened anti-tumor effect of MMC-loaded PPM. Another reason might be the slow-release effect of PPM on MMC. There are still some limitations in this study, such as the lack of EDX analysis and SEM analysis of polysaccharides, which require further research in the future.

\section{Conclusion}

This study successfully fabricates PPM containing MMC. The particle size of the MMC-loaded PPM was 30.10 $\pm 0.20 \mu \mathrm{m}$. In addition, the sustained release of MMCloaded PPM can promote the release of MMC at the target site, and decrease the dose into systemic circulation, 
thereby lowering the toxic efficacy on normal tissues. Furthermore, this anti-tumor experiment indicates that MMC-loaded PPM exhibits enhanced tumor inhibition. Therefore, PPM can be used as a novel and ideal drug carrier for tumor treatment.

\section{Acknowledgment}

This work was supported by the Natural Science Foundation of Zhejiang Province (No. LQ18H010004).

\section{Author Contributions}

Yuanbo $\mathrm{Wu}$ and Zhihao Yang are responsible for study design and data analysis. Jiangwei Ni and Jiandong Zhang contributed to the acquisition of data and performance of experiments. Kun Chen, Liangcheng Zheng and Jiandong Zhang are responsible for data interpretation and revision of the paper. Zhifeng He wrote the paper and performed the experiments. All authors contributed to data analysis, drafting or revising the article, have agreed on the journal to which the article will be submitted, gave final approval of the version to be published, and agree to be accountable for all aspects of the work.

\section{Disclosure}

The authors declare no conflicts of interest.

\section{References}

1. Li LX, Zhang B, Gong RZ. Insights into the role of tumor abnormal protein in early diagnosis of cancer: a prospective cohort study. Medicine. 2020;99:e19382. doi:10.1097/MD.0000000000019382

2. Mohammadinejad R, Dehshahri A, Sagar Madamsetty V, et al. In vivo gene delivery mediated by non-viral vectors for cancer therapy. J Control Release. 2020;325:249-275.

3. Lan F, Zhu M, Qi Q, Zhang Y, Liu Y. Prognostic value of serum tumor abnormal protein in gastric cancer patients. Mol Clin Oncol. 2016;5:216-220. doi:10.3892/mco.2016.877

4. Yang L, Li Y, Gou Y, et al. Improving tumor chemotherapy effect using an injectable self-healing hydrogel as drug carrier. Polym Chem. 2017;10:1-7.

5. Ashrafizadeh M, Zarrabi A, Hushmandi K, et al. Progress in natural compounds/siRNA co-delivery employing nanovehicles for cancer therapy. ACS Comb Sci. 2020;22:669-700. doi:10.1021/ acscombsci.0c00099

6. Zheng M, Hwang S, Snyder T, et al. Synthesis of Mitomycin C and decarbamoylmitomycin $\mathrm{C} \mathrm{N}(6)$ deoxyadenosine-adducts. Bioorg Chem. 2019;92:103280. doi:10.1016/j.bioorg.2019.103280

7. Radke PM, Bitrian E, Kaufman SC, Grajewski AL. A review of mitomycin use in ophthalmic surgery: clarification of safety standards for patients and hospital personnel. Curr Ophthalmol Rep. 2016;4:187-197. doi:10.1007/s40135-016-0111-2

8. Voskuilen CS, van de Kamp MW, Schuring N, et al. Radiation with concurrent radiosensitizing capecitabine tablets and single-dose mitomycin-C for muscle-invasive bladder cancer: a convenient alternative to 5-fluorouracil. Radiother Oncol. 2020;150:275-280. doi:10.1016/j.radonc.2020.07.057
9. Al-Otaibi WA, Alkhatib MH, Wali AN. Cytotoxicity and apoptosis enhancement in breast and cervical cancer cells upon coadministration of mitomycin $\mathrm{C}$ and essential oils in nanoemulsion formulations. Biomed Pharmacother. 2018;106:946-955. doi:10.1016/j. biopha.2018.07.041

10. McLeod HL. Cancer pharmacogenomics: early promise, but concerted effort needed. Science. 2013;339:1563-1566. doi:10.1126/ science. 1234139

11. Zhou Q, Zhang L, Yang T, Wu H. Stimuli-responsive polymeric micelles for drug delivery and cancer therapy. Int J Nanomedicine. 2018;13:2921-2942. doi:10.2147/IJN.S158696

12. $\mathrm{Xu} \mathrm{Y,} \mathrm{Hu} \mathrm{B,} \mathrm{Xu} \mathrm{J,} \mathrm{Wu} \mathrm{J,} \mathrm{Ye} \mathrm{B.} \mathrm{Preparation} \mathrm{of} \mathrm{biodegradable}$ polymeric nanocapsules for treatment of malignant tumor using coaxial capillary microfluidic device. Cancer Biother Radiopharm. 2020;35:570-580. doi:10.1089/cbr.2019.3412

13. Xi G, Liu W, Chen M, et al. Polysaccharide-based lotus seedpod surface-like porous microsphere with precise and controllable micromorphology for ultrarapid hemostasis. ACS Appl Mater Interfaces. 2019;11:46558-46571. doi:10.1021/acsami.9b17543

14. Gharbavi M, Johari B, Mousazadeh N, et al. Hybrid of niosomes and bio-synthesized selenium nanoparticles as a novel approach in drug delivery for cancer treatment. Mol Biol Rep. 2020;47:6517-6529. doi:10.1007/s11033-020-05704-z

15. Ashrafizadeh M, Hushmandi K, Rahmani Moghadam E, et al. Progress in delivery of siRNA-based therapeutics employing nano-vehicles for treatment of prostate cancer. Bioengineering. 2020;7:91. doi:10.3390/bioengineering7030091

16. Pawar H, Kamat SR, Choudhary PD. An overview of natural polysaccharides as biological macromolecules: their chemical modifications and pharmaceutical applications. Biol Med. 2014;7:224. doi:10.4172/0974-8369.1000224

17. He N, Shi X, Zhao Y, Tian L, Wang D, Yang X. Inhibitory effects and molecular mechanisms of selenium-containing tea polysaccharides on human breast cancer MCF-7 cells. J Agric Food Chem. 2013;61:579-588. doi:10.1021/jf3036929

18. Shu S, Sun L, Zhang X, Wu Z, Wang Z, Li C. Polysaccharidesbased polyelectrolyte nanoparticles as protein drugs delivery system. J Nanopart Res. 2011;13:3657-3670. doi:10.1007/ s11051-011-0284-4

19. Cheng JE, Si-Tong LU, Zhang HU, Si-Dong LI, Liao MN. Study on the preparation of the composite hemostatic microspheres of carboxymethyl chitosan/sodium alginate. Appl Chem Ind. 2018;47:501-504.

20. Yu HL, Feng ZQ, Zhang JJ, et al. The evaluation of proanthocyanidins/chitosan/lecithin microspheres as sustained drug delivery system. Biomed Res Int. 2018;2018:1-11. doi:10.1155/2018/ 6057348

21. Ahmadia E, Sadrjavadib K, Mohammadic G, Fattahid A. De-esterified tragacanth microspheres loaded into eudragit S-100 coated capsules for colon-targeted delivery. Iran J Pharm Res. 2018;17:470-479.

22. Moradi M, Abdolhosseini M, Zarrabi A, Johari B. A review on application of Nano-structures and Nano-objects with high potential for managing different aspects of bone malignancies. Nano-Struct Nano-Objects. 2019;19:100348. doi:10.1016/j.nanoso.2019.100348

23. Parhi R. Drug delivery applications of chitin and chitosan: a review. Environ Chem Lett. 2020;18:577-594. doi:10.1007/s10311-020-00963-5

24. Yu X, Pan Q, Zheng $\mathrm{Z}$, et al. $\mathrm{pH}$-responsive and porous vancomycin-loaded PLGA microspheres: evidence of controlled and sustained release for localized inflammation inhibition in vitro. RSC Adv. 2018;8:37424-37432. doi:10.1039/C8RA06659K

25. Kodama N, Murata Y, Asakawa A, et al. Maitake D-Fraction enhances antitumor effects and reduces immunosuppression by mitomycin-C in tumor-bearing mice. Nutrition. 2005;21:624-629. doi:10.1016/j.nut.2004.09.021 


\section{Publish your work in this journal}

Cancer Management and Research is an international, peer-reviewed open access journal focusing on cancer research and the optimal use of preventative and integrated treatment interventions to achieve improved outcomes, enhanced survival and quality of life for the cancer patient.
The manuscript management system is completely online and includes a very quick and fair peer-review system, which is all easy to use. Visit http://www.dovepress.com/testimonials.php to read real quotes from published authors.

Submit your manuscript here: https://www.dovepress.com/cancer-management-and-research-journa 